Mason, T. (1996) Seclusion and learning disabilities: Research and deduction. The British Journal of Developmental Disabilities, 42. 149-159.

Rangecroft, M. E. H., Tyrer, S. P. \& Berney, T. P. (1997) The use of seclusion and emergency medication in a hospital for people with learning disability. British journal of Psychiatry, I70. 273-277.

H. Sequeira, S. Halstead St Andrews Hospital, Northampton NNI 5DG

\section{Psychopathological syndromes and familial morbid risk of psychosis}

Sir: I wish to comment on the article by Van Os et al (1997). Before using the term "broad schizoaffective psychosis" the authors should consider the possibility that their original group of schizoaffective psychosis is already too broadly defined. Of the 150 patients with psychosis they diagnosed 65 as schizoaffective, giving a figure of $43 \%$ of consecutively admitted patients. When Brockington \& Leff (1976) looked at the diagnosis of schizoaffective psychosis in what would seem to be an identical setting, in 222 consecutively admitted patients they found an incidence of only eight cases diagnosed according to the same Research Diagnostic Criteria (RDC). An almost tenfold increase in the diagnosis needs an explanation.

The RDC acknowledges that schizoaffective disorders could be either variants of affective disorder or schizophrenia, and in fact lays down criteria to further subdivide schizoaffective psychosis into predominantly depressive or manic and predominantly schizophrenic. This distinction is important as Brockington et al (1980) have shown that schizomania is a variant of bipolar disorder and that schizodepressive disorder is often closely related to schizophrenia (Brockington et al, 1978). As no subdivision has been made this should compromise the results. It is possible therefore that the group of schizoaffective disorder which the authors compared with schizophrenia already had a large number of schizophrenics, hence the findings that schizophrenia familial risk was higher in this group. It would certainly be helpful if this information were available.

Adams, R. \&uylor, M. A. (1976) Catatonia, a prospective clinical study. Archives of General Psychiatry, 33. 579-581.

Brockington, I. F., Waimwright, S. \& Kendell, R. E. (1980) Manic patients with schizophrenic or paranoid symptoms. Psychological Medicine, 10. 73-83.
—, Kendell, R. E., Kellet, J. M., et al (1978) Trials of lithium, chlorpromazine and amitriptyline in schizoaffective patients. British Journal of Psychiatry, 133, 162-168.

- Lef, J. P. (1979) Schizoaffective psychosis: definition and incidence. Psychological Medicine, 9, 91-99.

Van Os, J., Marcelis, M. Sham, P., et al (1997) Psychopathological syndromes and familial morbid risk of psychosis. British journal of Psychiatry. 170. 24I-246.

A. Ryan Division of Mood Disorders, Sunnybrook Health Science Centre, 2075 Bayview Avenue, Toronto, Ontario M4N 3M5, Canada

Authors' reply: The likely reason for the higher rate of RDC schizoaffective disorder in our patients is that diagnoses were not only based on cross-sectional, but also on longitudinal (lifetime) psychopathological data. 'Pure' affective and schizophrenic states will become rarer with time as significant proportions of such patients will experience schizophrenic and affective symptoms respectively.

We agree that separate examination of schizodepressive and schizomanic cases would have been interesting and possibly revealing. The sample size, however, was too small to allow for such analyses.

While these diagnostic issues may be important, we would like to stress that the main findings of our paper involved effects that were independent of proband diagnosis.

J. Van Os, M. Marcelis Department of Psychiatry and Neuropsychology, PO BOX 616,6200 MD

Maastricht, The Netherlands

P. Sham, K. Gilvarry, R. Murray Institute of Psychiatry, London

P. Jones Mapperly Hospital, Nottingham

\section{Treatment, outcome and predictors of response in elderly depressed in-patients}

Sir: Heeren et al (1997) reported a naturalistic prospective study of treatment, outcome and predictors of response in elderly depressed in-patients in The Netherlands. Their findings showed a low percentage of full recovery $(33-45 \%)$, with a high rate of partial recovery $(43-66 \%)$, compared with previous studies. They concluded that a combination of inadequate treatment and therapeutic nihilism played a major role. Similar findings are paralleled in the adult service with failure to treat resistant depression adequately (Bridges et al, 1995).
However, there may be other reasons for such a low response in this study. The sample consisted of a wide variety of affective disorders, ranging from adjustment disorder to organic depression. Indeed, the study claimed to exclude patients with 'dementia'. How this was achieved is not clear and notably patients with Mini-Mental State Examination scores as low as five were recorded, with no evidence to suggest that this was a reversible cognitive impairment at follow-up. Thus this group of patients was an exceedingly heterogeneous mix, making it difficult to compare outcomes with previous studies.

If one assumes that the poor response is due to undertreatment, then this could be confirmed by comparing the two subgroups of patients, those treated with 'classic' antidepressants and 'modern' antidepressants. Undertreatment occurred much more frequently in the former group ( $82 v .36 \%$ ) and if their hypothesis is correct, should show a poorer prognosis. No comment was made regarding these data and it would be interesting to analyse this further.

Bridges, P. K., Hodzdiss, A. D. \& Malizia, A. L. (1995)

Practical management of treatment-resistant affective disorders. British journal of Psychiotry, 170. 436-440.

Heeren, T. J., Derkeen, P., Heycop Ton Ham, B. F. v., ot al (1997) Treatment, outcome and predictors of response in elderly depressed in-patients. British fournal of Psychiatry, 170 436-440.

G. Pinner, W. P. Bouman Department of Health Care of the Elderly, University Hospital, Nottingham NG7 2UH

Authors' reply: We thank Pinner \& Bouman for their critical comments and we agree with them that probably not only elderly depressives are the victims of therapeutic reticence. The first point concerns the heterogeneity of the population. The purpose of our study was to include as much as possible a 'normal' clinical population of elderly depressives and not a highly selected sample as usually occurs in clinical trials. Inclusion and exclusion were based on DSM-III-R criteria and not on the scores of the rating scales (these were used as measures of severity), so it could occur that one patient diagnosed with a recurrent major depressive episode had initially a score of five points on the MMSE. Although, unusual for a depressed patient, several factors such as motivation or physical illness may explain such a low score. 